\title{
Nonlinear Dynamics of Hyper-chaotic Complex Lu System
}

\author{
Jinping Jia, Fandi Zhang
}

School of Mathematics and Statistics, Tianshui Normal University, Tianshui 741001, China

Keywords: Fractional-order complex system; Bifurcation; Synchronization.

\begin{abstract}
We propose the fractional-order complex Lu system. Based on theoretical analysis and computer simulations, rich dynamics behavior of the system is investigated in detail. First of all, symmetry and the stability of equilibrium points are discussed. In addition, bifurcations with variation of different system parameters are discussed for diverse derivative orders. Chaotic attractors are further verified dynamical behavior of the system is very abundant.
\end{abstract}

\section{Introduction}

In the past decades, several researchers have focused their attention on the study of hyper chaotic systems with real variables in many fields and extensively studied due to wide scope potential applications (C.P. Li, 2006, P. Arena, R, 1997). On the other hand, fractional calculus which is considered as the generalized of the conventional calculus (namely, integer-order calculus) can be dated back to the 17th century. However, most of the existing works focus on fractional-order systems based on the state variables in real space, and complex systems are not involved. In fact, fractional-order hyper-chaotic systems which involving complex variables can be well describe the physics (J.G. Lu, 2006, G. M. Mahmoud, 2004).

Synchronization of systems is a very important nonlinear phenomenon. So far, many types of synchronization phenomenon have been proposed (G.M. Mahmoud, 2008, E. Roldab, 1993). From the point of view of secure communication, more unpredictable scaling factors can additionally enhance the security.

\section{Dynamics analysis of the fractional-order complex Lu system}

The dynamic behaviors of the Lu system written in the following from:

$$
\left\{\begin{array}{l}
\dot{y}_{1}=a\left(y_{1}-y_{2}\right)+y_{4} \\
\dot{y}_{2}=b y_{2}-y_{1} y_{3}+y_{4} \\
\dot{y}_{3}=y_{1} y_{2}-c y_{3} \\
\dot{y}_{4}=y_{1} y_{2}-d y_{4}
\end{array}\right.
$$

Where $a, b, c, d$ are positive parameters?

Suppose the state variable vector of the system (1) are defined in the complex field, and the derivative orders are fractional, thus hyper-chaotic fraction-order Lu system is defined as

$$
\left\{\begin{array}{l}
D_{*}^{q_{1}} y_{1}=a\left(y_{1}-y_{2}\right)+y_{4} \\
D_{*}^{q_{2}} y_{2}=b y_{2}-y_{1} y_{3}+y_{4} \\
D_{*}^{q_{3}} y_{3}=\frac{1}{2}\left(\bar{y}_{1} y_{2}+y_{1} \bar{y}_{2}\right)-c y_{3} \\
D_{*}^{q_{4}} y_{4}=\frac{1}{2}\left(\bar{y}_{1} y_{2}+y_{1} \bar{y}_{2}\right)-d y_{4}
\end{array}\right.
$$

Where $y=\left(y_{1}, y_{2}, y_{3}, y_{4}\right)^{\mathrm{T}}$ is the state vector, $y_{1}=x_{1}+j x_{2}, y_{2}=x_{3}+j x_{4}$ are complex functions, and $y_{3}=x_{5}, y_{4}=x_{6}$ are real functions and $j=\sqrt{-1}$. Based on the linearity of the Caputo differential 
operator, the real version of (2) reads:

$\left\{\begin{array}{l}D_{*}^{q_{1}} x_{1}=a\left(x_{3}-x_{1}\right)+x_{6} \\ D_{*}^{q_{1}} x_{2}=a\left(x_{4}-x_{2}\right)+x_{6} \\ D_{*}^{q_{2}} x_{3}=b x_{3}-x_{1} x_{5}+x_{6} \\ D_{*}^{q_{2}} x_{4}=b x_{4}-x_{2} x_{5}+x_{6} \\ D_{*}^{q_{3}} x_{5}=x_{1} x_{3}+x_{2} x_{4}-c x_{5} \\ D_{*}^{q_{4}} x_{6}=x_{1} x_{3}+x_{2} x_{4}-d x_{6}\end{array}\right.$

Symmetry

Firstly, symmetric about the $\mathrm{x}$-axis, which is invariant under the transformation

$S:\left(x_{1}, x_{2}, x_{3}, x_{4}, x_{5}, x_{6}\right) \rightarrow\left(-x_{1},-x_{2},-x_{3},-x_{4}, x_{5}, x_{6}\right)$,

Which permits the system (1) is invariant for all values of parameters with the transformation.

Equilibria and their stability

The equilibriums are:

$\left\{\begin{array}{l}\mathrm{E}_{1}(0,0,0,0,0,0) \\ \mathrm{E}_{\theta}(r \cos \theta, r \sin \theta, r \cos \theta, r \sin \theta, b, 0)\end{array}\right.$

Where $r=\sqrt{c b}$ and $\theta \in[0,2 \pi]$. It's obvious that the equilibrium $\mathrm{E}_{\theta}$ exists when $b c>0$.

The characteristic equation at equilibrium $\mathrm{E}_{1}$ is

$$
\begin{aligned}
& \lambda^{6}+(2 a-2 b+c+d) \lambda^{5}+\left(a^{2}-4 a b-2 a c-2 a d+b^{2}+c d\right) \lambda^{4} \\
& +\left(-2 a^{2} b+a^{2} c+a^{2} d+2 a b^{2}-4 a b c-4 a b d+2 a c d+b^{2} c+b^{2} d-2 b c d-2 b c-2 b d\right) \lambda^{3} \\
& +\left(a^{2} b^{2}+\left(2 a^{2} b^{2}-2 a^{2} b\right)(c+d)+a^{2} c d-4 a b c d+b^{2} c d\right) \lambda^{2} \\
& +\left(a^{2} b^{2} c+a^{2} b^{2} d-2 a^{2} b c d+2 a b^{2} c d\right) \lambda+a^{2} b^{2} c d=0
\end{aligned}
$$

The corresponding eigenvalues for the equilibrium point $E_{1}$ are $\lambda_{1}=-0.6, \lambda_{2,3}=-8.7761, \lambda_{4,5}=6.6761$

For the parameters $a=2.1, b=30, c=0.6$, which means $\mathrm{E}_{1}$ is unstable. The characteristic equation at equilibrium $\mathrm{E}_{\theta}$ is;

$$
\lambda(\lambda+a)\left[\lambda^{3}+(a+c) \lambda^{2}+b c \lambda+2 a c(b-a)\right]=0 .
$$

According to the Routh-Hurwitz conditions for fractional-order systems, $\mathrm{E}_{\theta}$ is stable for; $b(a+c)>2 a(b-a),(c \neq 0)$

When the parameters are $a=2.1, b=30, c=0.6$, the equilibrium point $\mathrm{E}_{\theta}$ is unstable.

\section{Dynamics of commensurate order Lu system}

We consider the same derivative orders in the system (3), that is $q_{1}=q_{2}=q_{3}=q$, which means the system (3) is a commensurate-order system.

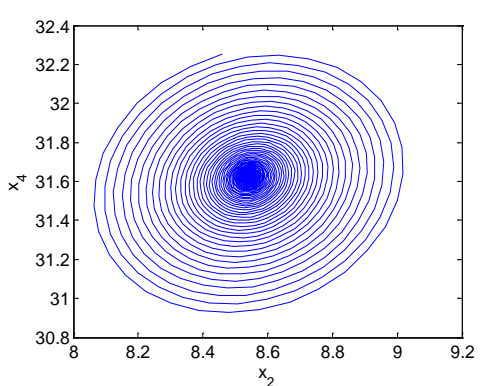

(a)

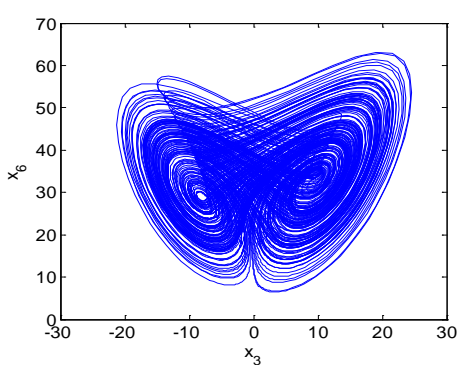

(b)

Figure 1. (a) The single-scroll attractor for $\mathrm{q}=0.81$; (b) The double-scroll attractor for $\mathrm{q}=0.98$ 


\section{Dynamics of incommensurate order Lu system}

Here we only consider typical differential order values and system parameters are taken as $(a, b, c, d)=(42,25,6,5)$, derivative orders are selected as $q_{1}=0.8, q_{3}=q_{4}=1, q_{2} \in(0.8,1)$;

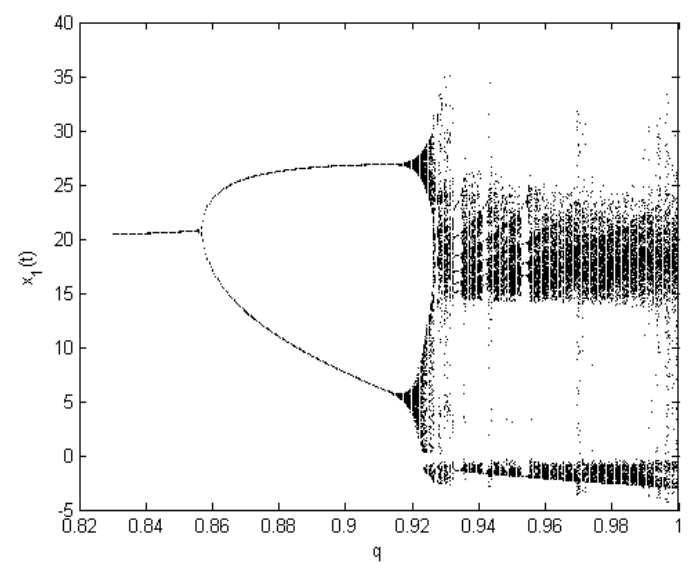

Figure 2. Dynamic behaviors of the fractional-order hyper-chaotic complex Lu system

When $q_{2}<0.86$ the system converges to a fixed point. Phase diagrams shown in Figs. 3 exhibit period-1 and chaotic attractors.

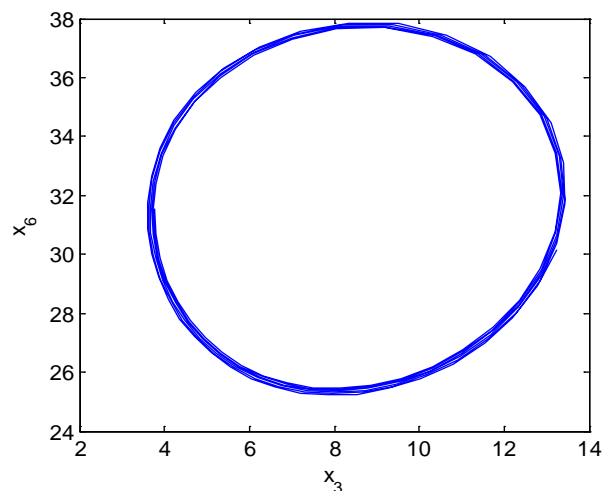

(a)

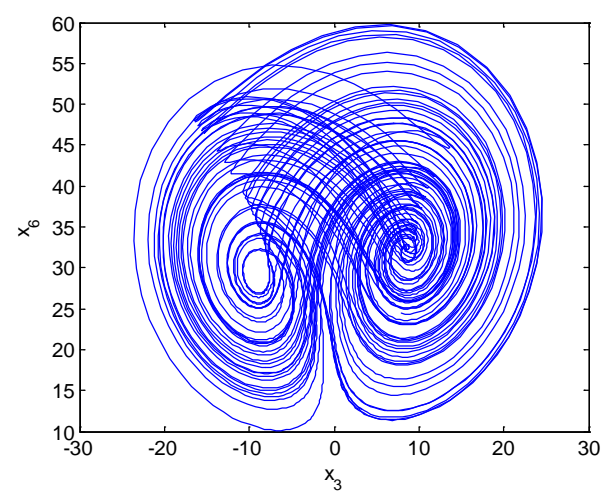

(b)

Figure 3. Phase diagrams for different values of the fractional order

\section{Conclusion}

In this paper, we proposed the fractional-order hyper-chaotic complex Lu system. The dynamics, chaotic attractors are discussed in details. Firstly, symmetry behaviors of the system are studied. Secondly, the stability of equilibrium points, bifurcations with varying the system parameters. 


\section{References}

C.P. Li, W.H. Deng, D. Xu. Chaos synchronization of the Chua system with a fractional order. Physica A Vol.360 (2006), No. 2, p.171-185.

E. Roldab, G.J. Devalcarcel, R. Vilaseca. Single-mode-laser phase dynamics. Phys, Rev. A Vol.48, (1993) No.1, p. 591-598.

J.G. Lu, G.R Chen. A note on the fractional-order Chen system. Chaos Solitions Fractals Vol.27 (2006), No.3, p. 685-688.

G. M. Mahmoud, T. Bountis. The dynamics of systems of complex nonlinear oscillators: a review. Int. J. Bifurcation chaos Vol. 14(2004) No.11, p. 3821-3846.

G.M. Mahmoud, M. A. Al-Kashif, A.A. Farghaly. Chaotic and hyper chaotic attractors of a complex nonlinear system. J. Phys. A: Math. Theor. Vol. 41 (2008) No.5, p. 055104.

P. Arena, R. Caponetto, L. Fortuna, D. Porto. Chaos in a fractional order Duffing system. In: Proc ECCTD. Budapest, (1997) p.1259-1262. 